\title{
HYDRODYNAMIC ANALYSES OF THE FLOW PATTERNS IN STIRRED VESSEL OF TWO-BLADED IMPELLER
}

\author{
Houssem Laidoudi \\ Laboratory of Science and Marine Engineering (LSIM), Faculty of Mechanical Engineering, \\ USTO-MB University, BP 1505, El -Menaouer, Oran 31000, Algeria \\ E-mail: houssem.laidoudi@univ-usto.dz
}

\begin{abstract}
In this paper, the governing equations of continuity and momentum subjected to suitable boundary conditions have been solved numerically to investigate the fluid flow in stirred vessel of two-bladed impeller. The numerical simulations have been carried out in three-dimensions for laminar flow. The studied fluid was considered Newtonian and incompressible. Our research studied the effects of geometrical configurations of the two-bladed impeller and its rotational speed on fluid patterns and mechanical power consumption. The innovative point in this paper is that the blades of the impeller contain three equal-sized holes of circular cross-section. The diameter of the hole $(d)$ to the impeller diameter $(D)$ gives the ratio $d / D$. the impeller speed is controlled by the Reynolds number $(R e)$. The obtained results have been illustrated and discussed for the range of following governing parameters: $d / D=0$ to 0.4 and $R e=1$ to 300 . The results showed that the studied parameters have significant effects on fluid flow and consumption power and the perforated blades of ratio $d / D=0.133$ is more efficient than plan blades. Also, a new correlation is proposed to describe the consumption power as function of $d / D$ and $R e$.
\end{abstract}

Keywords: Stirred vessel, two-bladed impeller, Newtonian fluid, perforated blade, laminar regime.

\section{Introduction}

Many mechanical applications involve a processing step called mixing liquids. Generally, this process is achieving by using a stirred tank in which there is a rotating impeller of different shapes. The obtained mixed liquid is the principal material that is used for preparing the pharmaceutical, petroleum, cosmetic and eating products. The mechanical agitation could be also encountered in many other fields of industrial applications such as applications of food treatment, metallurgies, mass transfer, hydrology, heat transfer and so on. The previous researches in this field have indicated that the quality of mixed liquid depends essentially on the geometrical configurations of agitator (the form of impeller as well as the stirred vessel) as seen in (RamírezMuñoz et al. 2016; Fort 2011; Fort et al. 2013), the fluid parameters (density, viscosity...) (Kazemzadeh et al. 2017; Ameur 2018; Moucha et al. 2003) and operating mode (rotation speed of impeller, with or without thermal treatment) (Alic 2013; Rajasekaran et al. 2018; Driss et al. 2011). On the whole, the size of the agitator and its variety depends extremely on the application. 
Recently, remarkable numbers of experimental and numerical researches have been devoted to study the flow patterns in a stirred tank. All in all, the main purpose of all these works is to design an efficient agitator (increasing the mixing quality while decreasing the mixing time). Energetically, the mechanical agitator is an energetic convertor that can convert the mechanical energy to kinematic energy, describing hence the motion of fluid inside the vessel. The fluid motion in agitated tank characterizes the mixing quality by looking into the nature of generated vortices which are created by the impeller blades. It can be concluded that the analyses of flow patterns in agitated vessel is a mandatory step before estimating the final shape of an efficient impeller.

Bouzit et al. (2006) numerically investigated three-dimensional simulations of the flow in stirred vessel. The agitator has a two-bladed impeller. The research has examined the effects of blade dimension (diameter and height) and its rotation on the global velocity profiles. The value of rotational speed has been determined for the value 20 of Reynolds number. Hadjeb et al. (2019) carried out a numerical simulation in order to give a new design of a two-bladed impeller. The studied geometries were a combination of ordinal two-bladed agitator with a helical screw agitator while the vessel was kept cylindrical with flat bottom. The computational results are limited only for two values of Reynolds number $(R e=10$ and 20$)$. The governing equations of CFD model have been solved in steady-state for laminar flow. The investigation have proofed that the new concept of impeller is more efficient than the simple impeller. Ameur et al. (2018a) numerically studied a new form of two-bladed impeller in cylindrical tank. The impeller and the vessel heights are similar. The blades of the impeller were supposed to be curved. It was concluded that the curved blades are more efficient than the straight blades. (Liu et al. 2015) studied a mixing liquid in cylindrical tank by novel large-double-bladed impeller. The vessel bottom is curved. The novelty in this geometry is that the impeller involves some rectangular holes. This kind of agitators are using in rapid reactions. Hashimoto et al. (2009) experimentally investigated the isolating region in mixing fluid in cylindrical container. The impeller is twoblade paddle. The bottom of the container is flat. Ameur and Bouzit (2013) carried out a numerical simulation of two-bladed impeller in cylindrical tank of flat bottom. The flow used in this work was non-Newtonian power-law. The apparent dynamic viscosity of the fluids was described by the Oswald model. The research examined the ratio between the vessel and impeller heights. The correlation describing the power consumption versus the geometrical configuration and fluidic proprieties was given.

In order to increase the performance of mechanical agitation, there are many researchers modified the form of the stirred vessel, some baffles were added vertically to the container (Ammar et al. 2011; Vilard and Verdone 2020; Tamburini et al. 2012; Tacay and Payunescu 2000; Vakili et al. 2009). Foukrach et al. (2019) changed the arrangement of baffle from the vertical to horizontal and Kamla et al. (2017) inclined the vertical baffles in cylindrical tank by inclination angle. Other category of researchers used a cylindrical vessel of curved bottom inside of flat bottom and it was found a significant impact on impeller performance (Ameur et al. 2018b and Kang et al. 2019). Ameur (2016) numerically studied a Bingham fluid in mechanical agitator of Scaba 6SRGT impeller and different form of the stirred vessel (cylindrical with fat bottom, cylindrical with curved bottom and completely spherical). The results showed that the spherical configuration generated a uniform rotational flow with less consumption energy.

Some modifications haves been also oriented to the form of impeller for the same reason. Ameur et al. (2019 and 2020) introduced the effects of cutting impeller (6SRGT) on overall fluid motion. (Luan et al. 2017) added the effects of eccentricity arrangement and clearance distance on 6PBT impeller in cylindrical vessel of flat bottom. The pseudoplastic fluids were used for this work. Martinez-Delgadillo et al. (2019) studied the radial turbine by modifying the blade of turbine from straight blade to $\mathrm{U}$ and $\mathrm{V}$ grooved blade. At the end of the research, it was concluded that the impeller of $\mathrm{V}$-grooves shape had the more influence on the dissipation of kinematic 
energy. Su et al. (2018) modified the Rushton impeller by adding two circular covering-plates placed on upper and lower parts of the impeller.

From the bibliographic analyses of previous works in this field, it can be concluded that the shape of the impeller is a critical factor that can improve the performance of mechanical agitation. Also, there is no work focused on the geometrical modification of two-bladed impeller by creating holes on the blades. Therefore, we decided through this research to examine the effects of perforated blades of the paddled impeller on the flow patterns and the consumption power. However, the blade of impeller contains three equal-sized holes. This work shows in detail all kinds of generated vortices during the rotation of the impeller. Also, it describes quantitatively the consumption power of the impeller as function of rotating speed.

\section{Description of mixing system and the mathematical formulation}

Fig. 1 reflects the geometrical configuration of studied mixing system. The system consists of a cylindrical tank of flat bottom and without baffles. The diameter and the height of tank are the same in size and they are given by the notations $(T)$ and $(H)$, respectively. A two-bladed paddled impeller of diameter $(D)$ and the height $(h)$ is placed in the centre of tank Fig.1 (a). The gap distance between the flat base of tank and the rotating impeller is given by the clearance ratio $c / T$ $=0.033$. The diameter of the impeller to the diameter of the tank defines the ratio $D / T=0.5$. The thickness of the blades and the diameter of the impeller shaft are given by the ratios $t / T=0.008$ and $d_{S} / T=0.016$, respectively. Fig. 1 (b) shows the new geometrical modifications of the blades. Three holes of circular cross-section are vertically arranged in the center of each blade. The separating distance between the holes is defined by dividing the impeller height $(h)$ by the value 4. The diameter of the holes is defined by ratio $d / D=0,0.066,0.133,0.2,0.266$ and 0.4 . It is very important to mention here that the stirred tank is supposed to be completely filled with mixing liquid. All previous geometrical characteristics of the mixing system are summarized in Table 1.

The fluid used for present mixing system is Newtonian and impressible with following characteristics: the dynamic viscosity $\eta=0.00089(\mathrm{~Pa} \mathrm{~s})$ and the density $\rho=997\left(\mathrm{Kg} / \mathrm{m}^{3}\right)$. The effect of temperature on the working fluid is negligible.

\begin{tabular}{|l|c|c|c|c|c|c|}
\hline$T[\mathrm{~mm}]$ & $\mathrm{H} / \mathrm{T}$ & $\mathrm{D} / \mathrm{T}$ & $\mathrm{c} / \mathrm{T}$ & $\mathrm{h} / \mathrm{T}$ & $\mathrm{t} / \mathrm{T}$ & $\mathrm{d}_{\mathrm{s}} / \mathrm{T}$ \\
\hline 300 & 1 & 0.5 & 0.033 & 0.966 & 0.008 & 0.016 \\
\hline
\end{tabular}

Table 1. Geometrical parameters of present mixing agitator 

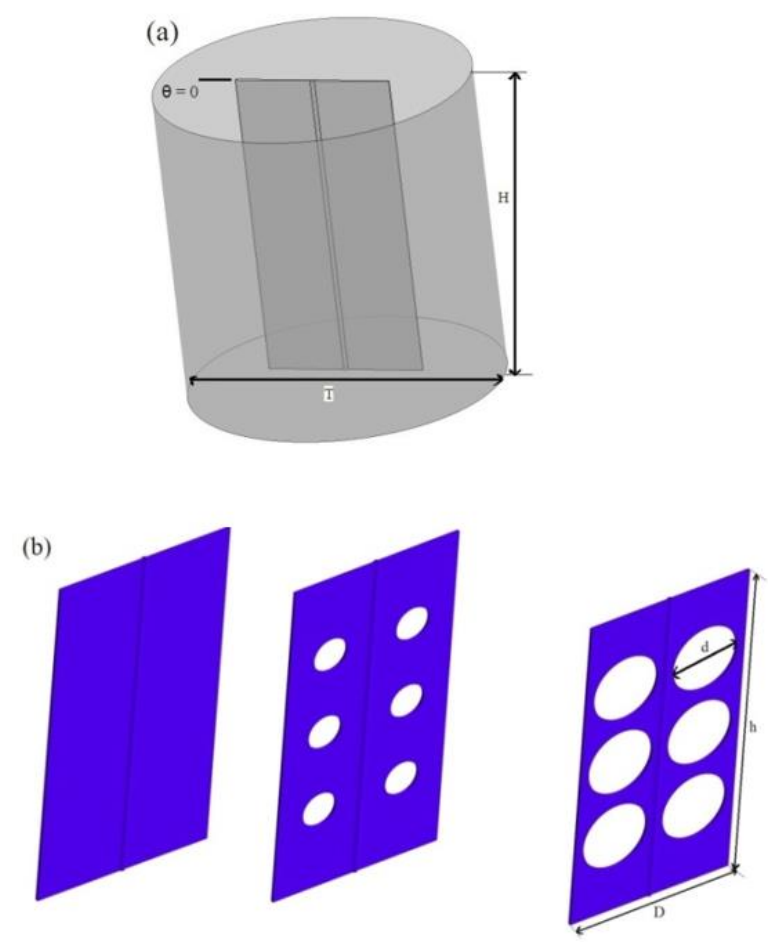

Fig. 1. Mixing system, (a) the stirred vessel with ordinal impeller, (b) the pierced blades with different diameters

In present work, the rotational speed of the impeller is controlled by the Reynolds number which is defined by the expression:

$$
R e=\frac{\rho N D^{2}}{\eta}
$$

where $(N)$ is the rotational speed of the impeller and $(D)$ is the diameter of the impeller.

The cylindrical coordinate system is used to representing the obtained results. Therefore, the dimensionless coordinates of the stirred vessel are expressed in cylindrical coordinate system (radial direction and axial direction) by the following:

$$
\begin{aligned}
R^{*} & =\frac{2 R}{T} \\
Z^{*} & =\frac{Z}{H}
\end{aligned}
$$

where $R$ is the radial coordinate of cylindrical tank and the $Z$ is the axial toward the $\mathrm{z}$ - direction (the height of tank).

In cylindrical coordinate system, the flow velocity has three components. $V_{\theta}, V_{r}$ and $V_{Z}$ are the tangential, radial and axial velocities respectively. The dimensionless values of the velocity components are written as: 


$$
\begin{aligned}
V_{\theta}^{*}=\frac{V_{\theta}}{V_{r o t}}, V_{r}^{*} & =\frac{V_{r}}{V_{r o t}}, V_{z}^{*}=\frac{V_{z}}{V_{r o t}} \\
V_{r o t} & =\pi N D
\end{aligned}
$$

When the impeller rotates in filled tank with real fluid, two kinds of forces are exerted on the blades of the impeller. These forces are pressure and the viscous forces. Therefore, a sufficient mechanical power is required from the impeller to drag the fluid. In this paper, the mechanical power is defined by the dimensionless number called the power number and it is expressed as:

$$
N p=P / \rho N^{3} D^{5}
$$

where $P$ is the required mechanical power which calculated by:

$$
P=2 \pi \times N \times T r
$$

where $\operatorname{Tr}$ is the torque determined from the impeller shaft.

The appropriate boundary conditions for the present investigation are: no-slip condition is considered for the walls of the tank and the impeller, whereas the slip condition is considered at the top of the tank. However, the impeller rotates with rotational velocity of $N$. these boundary conditions are similar to the previous work of (Laidoudi and Ameur 2020).

\section{Numerical approach}

The present investigation is carried out by using the commercial package of ANSYS-CFX. This CFD tool converts the governing partial differential equations of continuity and momentum into matrix systems then it solves them by using the finite-volume method. To carry out this task, the present mixing domain is divided into small cell elements called volumes of control. The generation of mixing system with suitable mesh is perfectly accomplished with the help of software program (Gambit). Tetrahedral unstructured mesh with non-uniform dissipation of cellular elements is generated for meshing the volume space of the domain. On other hand, the structural mesh with uniform distribution is generated only for meshing the surfaces of the impeller and tank. Fig. 2 shows the studied domain with its mesh. The present model of mixing system comprises 900393 cells. This value was taken into account after the satisfactory analyses of grid independency test which is presented in Table 2. Indeed, Table 2 shows the effect of number of cells on the computational results of average velocity of the flow in the entire tank for $R e=50$ and $d / D=0.266$. It is clear that the effect of additional cells on the average velocity is almost negligible over the value 900393. Therefore, the mesh M2 is satisfactory for the present computations. The obtained results of present investigation are considered when the error of convergence criteria becomes less than $10^{-7}$ for the continuity and momentum equations. 


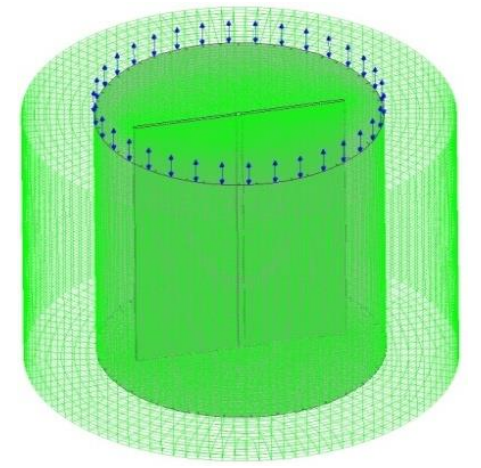

Fig. 2. Mesh for present mixing system

\begin{tabular}{|c|c|c|}
\hline Mesh & Number of cells & Average velocity \\
\hline M1 & 100738 & 0.61232 \\
\hline M2 & 900393 & 0.68125 \\
\hline M3 & 1809877 & 0.68045 \\
\hline
\end{tabular}

Table 2. Details on the grid independency test

In order to simulate a rotational flow inside a container with a rotating impeller, the multiple reference frame (MRF) technique was employed for this objective. This approach is commonly used by many previous works such as Foukrach et al. (2019), Ameur (2019) and so on. The principle of this method is based on dividing the entire domain into two principal parts; the inner rotating domain which involves the impeller and the outer stationary domain which is surrounded by the walls of the vessel.

This section is devoted also to proof the accuracy of our adopted numerical approach for the determination of predictive results. This comprehensive study touches the numerical method, chose of grid element size and suitable boundary conditions. For this purpose, we have repeated the same experimental investigation of Youcefi et al. (1993) and under the same initial conditions. Our numerical results with the prior experimental results are depicted together in Fig. 3 (a). The figure shows the variation of tangential velocity along the radius $R^{*}$ of the angular position $\theta=$ $9 \theta^{\circ}$ at the height $Z^{*}=0.87$ for a two-bladed impeller of $T=400 \mathrm{~mm}$ rotating with value 4 of $\mathrm{Re}$. A good concordance is shown between the two plotted graphs. Another comparison is also realized with numerical correlation of Ameur and Bouzit (2013) Fig. 3 (b). The figure shows the variation of power consumption number with $R e$ number for two-bladed agitator of $T=300 \mathrm{~mm}$. A good agreement is observed between the results, too. 

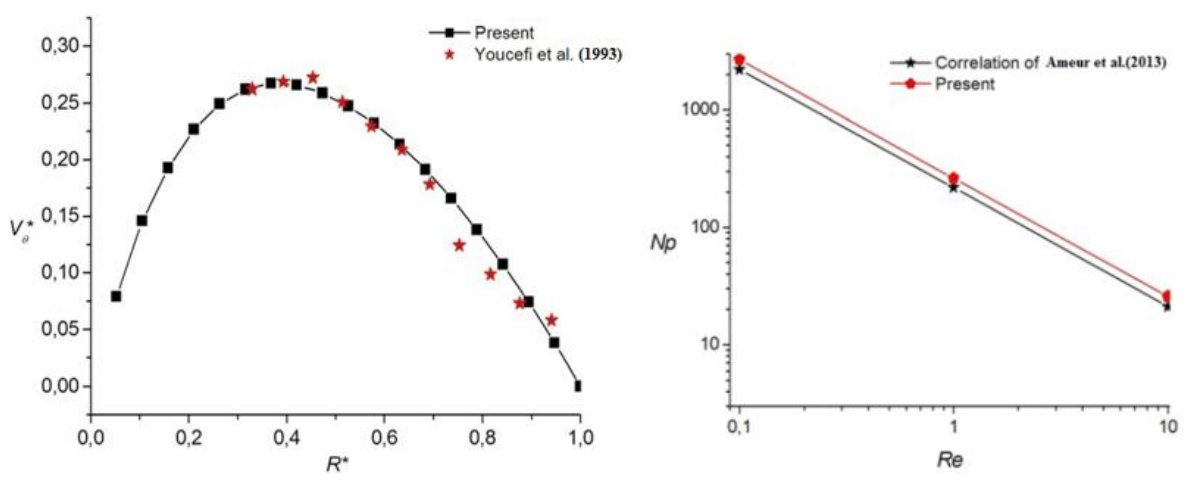

Fig. 3. Comparison results, (a) dimensionless tangential velocity versus dimensionless radius of thank for $\operatorname{Re}=4$ and $\theta=90^{\circ}$, (b) power number versus Reynolds number

\section{Results and discussion}

Numerical investigations are carried out to correctly predict the flow patterns inside a stirred vessel. The fluid motion is induced by the rotation of two-blade paddled impeller. Some new geometrical modifications are addressed to the blades of the impeller. These modifications consist of piercing the blades with three equal-sized holes of circular cross-section. On whole, the present work studies the effects of hole diameter and rotational speed of impeller on the flow patterns and mechanical power consumption. The rotational speed of the impeller is controlled by the Reynolds number meanwhile, the diameter of holes is controlled by the ratio $d / D$.

Fig. 4 depicts the three-dimensional structure of flow patterns for different values of Reynolds number (a) $R e=30$ and (b) $R e=300$ for $d / D=0$. It is shown that the flow in stirred vessel of two-bladed impeller produces two forms of flow structures based on the value of rotational speed of impeller. The first structure is produced for low value of Reynolds number and it consists of single block of flow with two parallel vortices in the gap spaces between the blades and the vessel walls (at the angle $\theta=0^{\circ}$ and $90^{\circ}$ ). However, the second one is formed over the critical values of Reynolds number of $R e_{c}=250$. The new structural form consists of dividing the vertical look of flow into two parts. Furthermore, the second structure suppresses completely the three-dimensional vortices between the blades and the walls of the container. 
(a)

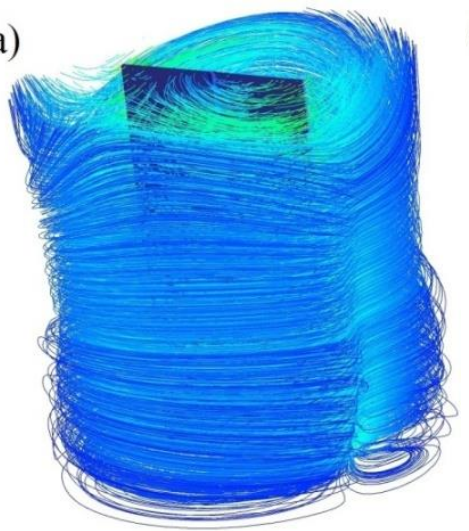

(b)

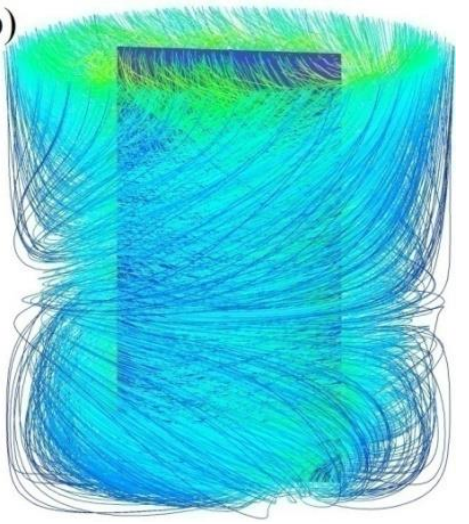

Fig. 4. Tree-dimensional streamlines for simple impeller with different $R e$, (a) $R e=30$, (b) $R e=$ 300 for $d / D=0$

Fig.5 shows two-dimensional streamlines at axial positions $Z^{*}=0.2$ and 0.66 for different values of the ratio $d / D$ and Reynolds number. It is evident that a two-dimensional vortex is seen between the blade of the impeller and the tank surface. The size of the vortex gradually increases as we move toward the tank bottom. Also, the increase in the hole diameter increases little the size of the bubble. In the contrary, the increase in the value of Reynolds number suppresses the counter-rotating region that is between the blade and the tank wall. The exact value of Reynolds number responsible for the suppression of this kind of vortex is almost 250 for all studied values of $d / D$.

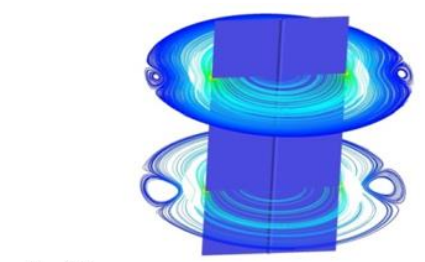

$\operatorname{Re}=30$

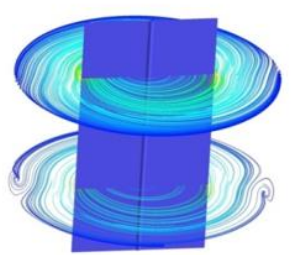

$R e=200$
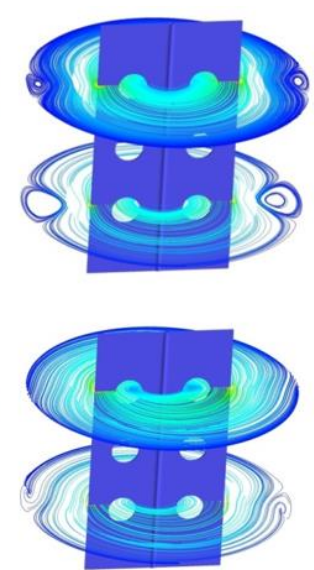
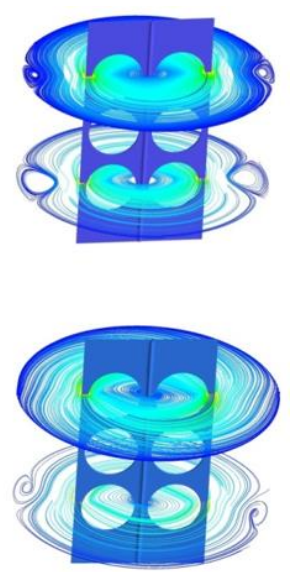

$$
d / D=0
$$$$
d / D=0.133
$$

$d / D=0.4$

Fig. 5. Streamlines at axial direction $Z^{*}=0.2$ and 0.66 for $R e=30,200$ and $d / D=0,0.133$ and 0.4

Fig.6 depicts the dimensionless velocity contours at the axial position $Z^{*}=0.55$ and at fixed value of $R e=100$ for different values of ratio $d / D=0,0.066,0.266$ and 0.4 . It can be observed 
that for all studied geometries, the maximum value of velocity is at tip of the blades. Furthermore, as we move from the ratio $d / D=0$ to 0.0166 the velocity increases little and the stagnation region around the impeller shift decreases describing hence increase of the zone of well-mixing region inside the vessel. On other hand, the velocity decreases and the stagnation region around the shaft increases again when the radio $d / D$ continue to increase from 0.066 to 0.4 .
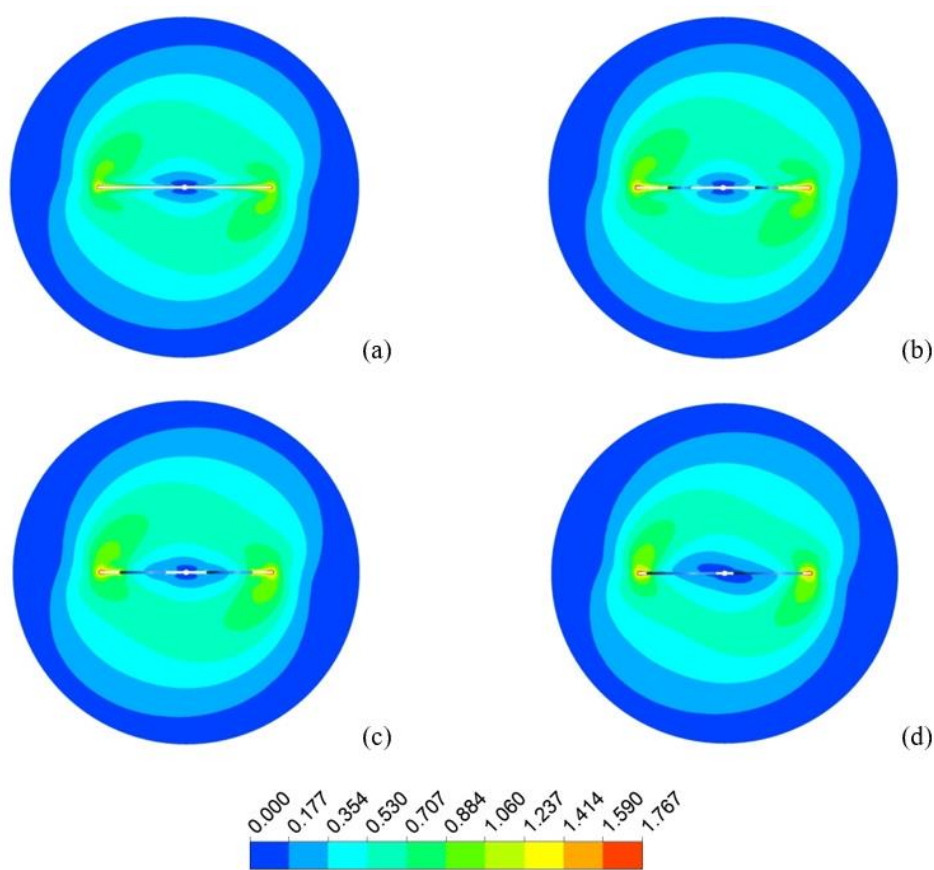

Fig. 6. Dimensionless velocity contours at $Z^{*}=0.55$ and $R e=100$, for (a) $d / D=0$, (b) $d / D=$ 0.066 , (c) $d / D=0.266$ and (d) $d / D=0.4$

Fig. 7 shows the vertical distribution of two-dimensional streamlines at the vertical section corresponding to the angular position $90^{\circ}$. The streamlines are symmetrical for both sides of the vessel. Therefore, only one side is represented. The figure determines the effects of Reynolds number and ratio $d / D$ on the streamlines. It is very important to clarifying that during a completed rotation of the impeller, the blades drag the fluid in same direction of the impeller rotation. As consequence, an instant vacuum is created at the vertical section $90^{\circ}$ of the tank. Accordingly, the fluid rapidly moves toward these regions in order to fill them. Consequently, two counterrotating regions are seen to be formed in this section. The size of the bubbles increases in both directions with increasing the value of Reynolds number. Also, the tension of follow patterns increases with $R e$ hinting the size of well-mixing region becomes important with $R e$. the effects of hole diameter on flow patterns in this case is almost negligible for both values of $R e$.

Fig. 8 shows tow-dimensional streamlines at the vertical section of the middle of the radius $R^{*}$ corresponding to angle $\theta=0^{\circ}$ for $R e=100,300$ and $d / D=0,0.4$. The figure indicates that over the critical value of Reynolds number $(R e=250)$, there is apparition of a new sort of recirculation region behind the impeller blades on the bottom of the tank. The size of the vortex decreases in axial direction with the presence of hole on the blade. 

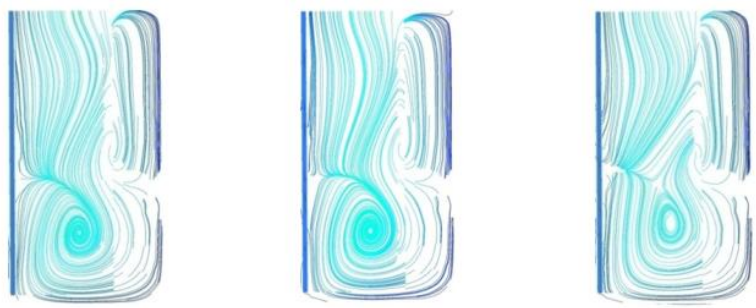

$R e=30$
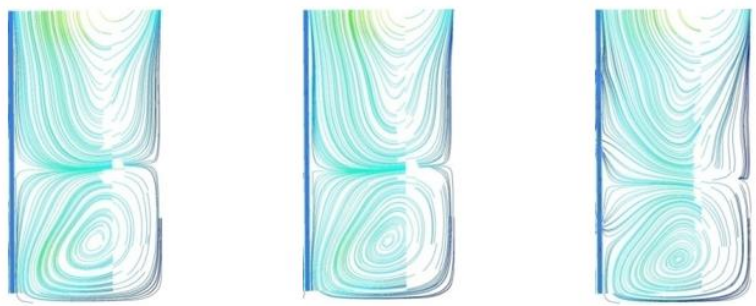

$R e=300$

$$
d / D=0 \quad d / D=0.266 \quad d / D=0.4
$$

Fig. 7. Two-dimensional streamlines along the middle tank at $\theta=90^{\circ}$ for $\operatorname{Re}=30,300$ and $d / D$ $=0, \mathrm{~d} / \mathrm{D}=0.266$ and $\mathrm{d} / \mathrm{D}=0.4$
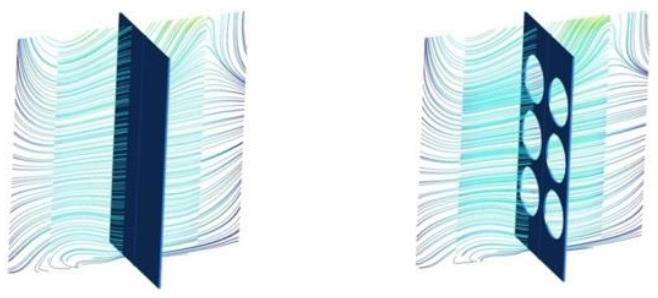

$\operatorname{Re}=100$
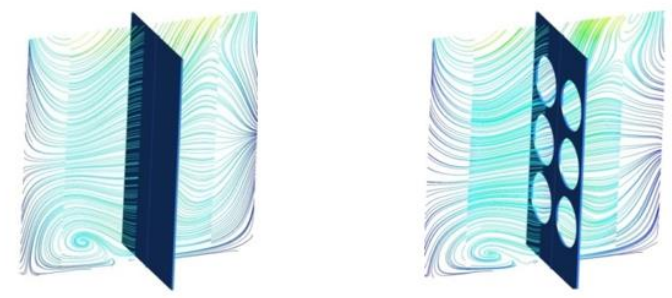

$\mathrm{Re}=300$

$$
\mathrm{d} / \mathrm{D}=0 \quad \mathrm{~d} / \mathrm{D}=0.4
$$

Fig. 8. Vertical streamlines along the middle blade section at $R^{*}=0.5$ and $\theta=0^{\circ}$ for $R e=100$, 300 and $d / D=0,0.4$ 
The streamlines for the vertical section of the angular position of $\theta=0^{\circ}$ for $R e=100$ and for different values of ratio $d / D$ are depicted in Fig. 9. It is clear that the flow patterns are shown to be divided into two parts at the axial position $\mathrm{Z}^{*}=0.33$. Furthermore, the holes of the blade create some counter-rotating zones in the holes for the ratio $d / D=0.133$ and 0.4 .
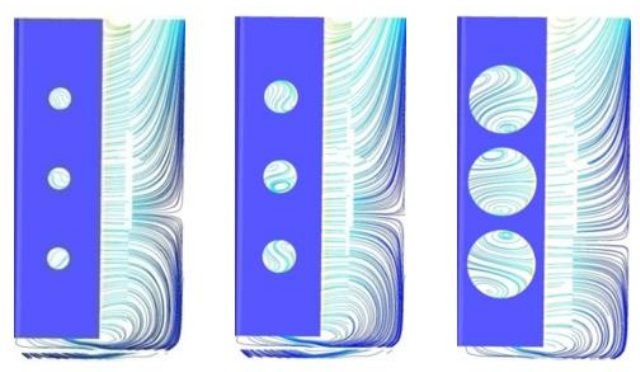

$$
d / D=0.066
$$

$$
d / D=0.133
$$

$d / D=0.4$

Fig.9. Two-dimensional streamlines along the middle tank at $\theta=0^{\circ}$ for $R e=100$ and $d / D=0$,

$$
d / D=0.066,0.133 \text { and } 0.4
$$

Fig. 10 represents the local values of tangential velocity along the radius $\left(R^{*}\right)$ at the angular position $\left(\theta^{\circ}=0\right)$ and at the dimensionless height $Z^{*}=0.27$. At this level, the displacement along the radius ( 0 to 1 ) crosses horizontally the circular hole of blade in the middle. The velocity profiles are plotted to show the effect of the ratio $(d / D)$ on it at fixed Reynolds number $R e=50$. For all geometrical cases. The highest local value of the tangential velocity is detected at the tip of blade. It is also shown that the pierced blade increases tightly the tangential velocity at the blade tip. Interesting variation of velocity distribution is seen along the blade diameter. The gradual increase in ratio $d / D$ causes a progressive reduction of tangential velocity due to the presence of hole on blade and the diameter of the hole increases with increasing the ratio $d / D$.

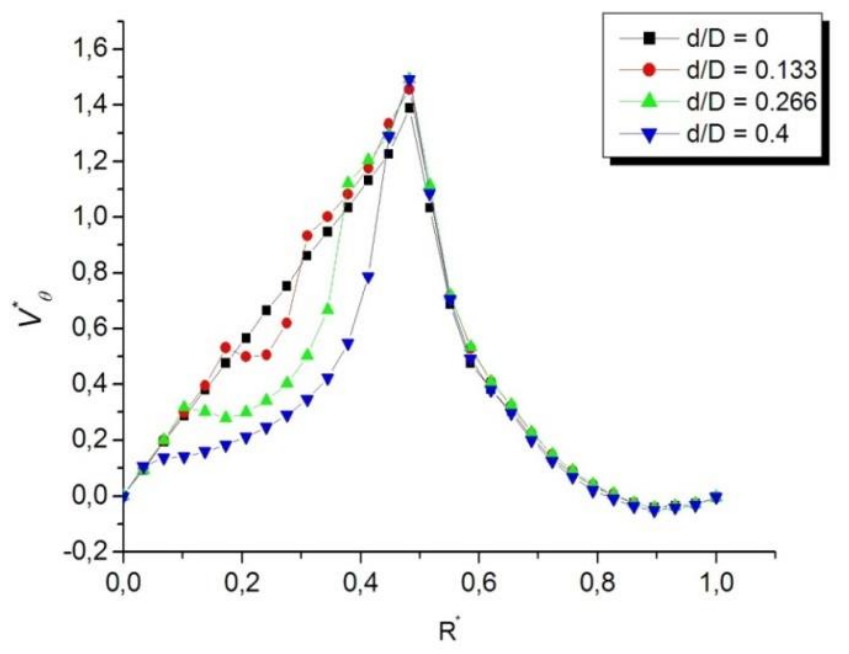

Fig.10. Tangential velocity along the radius of angular position $\theta=\theta^{\circ}$ at the axial position $Z^{*}=$ 0.27 for $R e=80$ and $d / D=0,0.133,0.266$ and 0.4 
Fig. 11 plots the dimensionless velocity profiles of tangential component along the radius $R^{*}$ for different values of ratio $d / D$ at the angular position $\theta=9 \theta^{\circ}$ and at dimensionless height of $Z^{*}=0.51$ for Reynolds number of $R e=80$. It is evident that the highest value of the velocity is between 0.3 and 0.4 of the radius $R^{*}$. Clearly, this region is well-dragged by the impeller. Accordingly, it produces speed displacement of fluid particles. It is also shown that increase in ratio $d / D$ enhances the tangential velocity up to the value 0.266 then the velocity decreases again. Finally, it can be concluded that the piercing blade of the ratio $d / D=0.133$ generates a tangential velocity better than simple blade $(d / D=0)$.

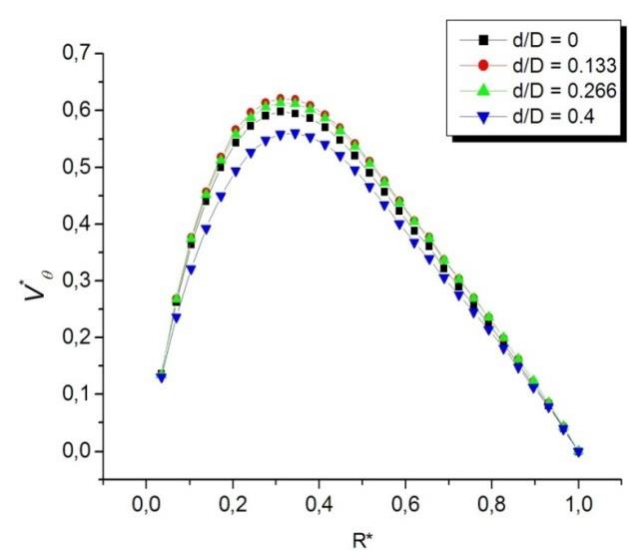

Fig. 11. Tangential velocity along the radius of angular position $\theta=9 \theta^{\circ}$ at the axial position $Z^{*}=0.51$ for $R e=80$ and $d / D=0,0.133,0.266$ and 0.4

Fig. 12 presents the local axial velocity in dimensionless form along the height of the tank at the radial position 0.66 of the angle $\theta=9 \theta^{\circ}$. The velocity profiles are potted as function of the ratio $d / D$ at fixed $R e=80$. The negative value of velocity shows the opposite direction of the flow due to vortex. It can be seen that along the axial direction starting from 0.1 to 0.42 and from 0.9 to 1 , the piercing blade with ratio $d / D$ generates an axial velocity more than simple blade.

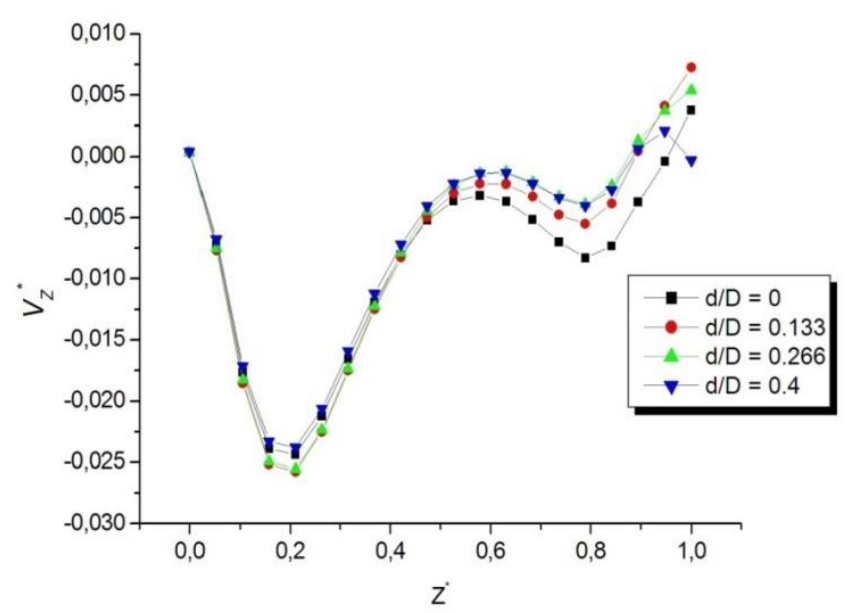

Fig. 12. Axial velocity along the axial direction at $R^{*}=0.66$ of $\theta=9 \theta^{\circ}$ for $R e=80$ and $d / D=0$, $0.133,0.266$ and 0.4 
Fig. 13 summarizes the variation of dimensionless average velocity in the stirred tank as function of the studied ratio $d / D$ for different values of Reynolds number. For all geometrical cases the increase in the value of Reynolds number increases the average velocity which produces well-mixed liquid. On other hand, for all studied values of Reynolds number, the increase in the value of ratio $d / D$ increases the average velocity up to the value 0.133 then it decreases again indicating the optimal value of ratio $d / D$ responsible for the best mixing factor.

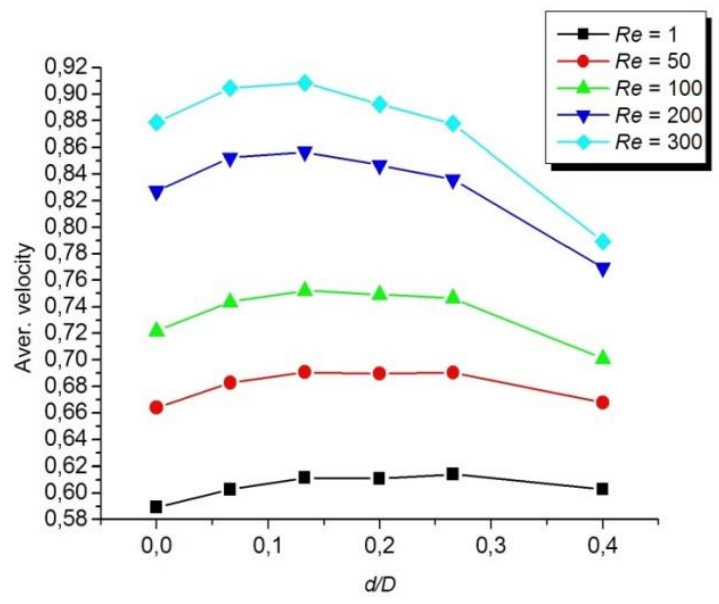

Fig.13. Variation of average velocity in stirred tank versus the ratio $d / D=0$ to 0.4 for different values of Reynolds number

Fig. 14 shows the variation of power number versus the ratio $d / D$ for different values of Reynolds number. It is clear that increase in value of Reynolds number influences negatively on the power number this is due to the viscous force which decreases with increasing the velocity. Also, the effect of the geometrical ratio on the power number is almost negligible for all values of Reynolds number. Furthermore, it is observed that the effect of Reynolds number on power number becomes negligible when Reynolds number passes the value 200.

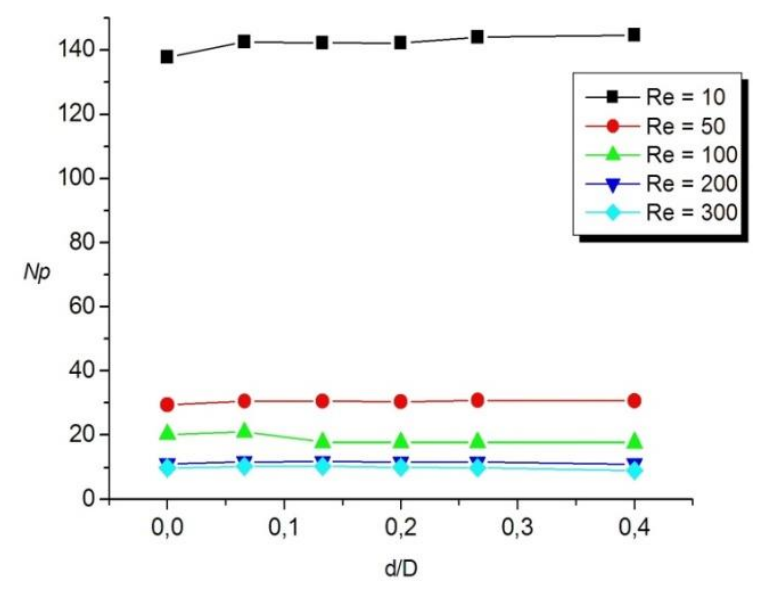

Fig.14. Power number versus d/D for different values of Re 
At the end of this work, a new correlation is proposed for describing the evolutions of power number as function of Reynolds number and the ratio $d / D$.

$$
N p=14.90+235.23 e^{\left(\left(\frac{-d}{D}\right) / 13.28-R e / 16.17\right)}
$$

\section{Conclusion}

The computational fluid dynamics (CFD) is employed to numerically investigate the fluid flow inside the stirred vessel of two-bladed impeller. Some new geometrical modifications are addressed to the impeller blades in order to increase the mixing performance. Three circular holes are vertically pierced in the middle of blades. On the whole, this work examines the effects of hole diameter and the impeller speed on the flow velocity and power number. The results are obtained for the range of following initial conditions: $R e=1$ to 300 and $d / D=0$ to 0.4 . The validation test showed a good agreement with the prior results in the same field. The present results proofed that the blades that have the holes of the ratio $d / D=0.133$ is the most efficient in term of increasing the mixing velocity and decreasing the power number. The present work shows also the evolutions of flow patterns versus Reynolds number and geometrical configuration. It was observed that when Reynolds number is less than its critical value $\left(R e_{c}=250\right)$ the flow seems single block with two three-dimensional vortices which are in the spacing between the blades and the vessel walls. On other hand, when Reynolds number is over the critical value, there is a suppression of the lateral vertices and the flow divided vertically into two parts.

\section{References}

Ameur H (2016). Agitation of yield stress fluids in different vessel shapes. Engineering Science and Technology. 19: 189-196.

Ameur H (2018). Modifications in the Rushton turbine for mixing viscoplastic fluids. Journal of Food Engineering, 233: 117-125.

Ameur H (2019). Some modifications in the Scaba 6SRGT impeller to enhance the mixing characteristics of Hershel-Bulkley fluids. Food and Bioproducts Processing, 117: 302-309.

Ameur H, Bouzit M (2013). Power consumption for stirring shear thinning fluids by two-blade impeller. Energy, 50: 326-332.

Ameur H, Kamla Y, Sahel D (2018a). Optimization of the operating and design conditions to reduce the power consumption in a vessel stirred by a paddle impeller. Periodica Polytechnica Mechanical Engineering, 62: 12-319.

Ameur H, Kamla Y, Sahel D (2018b). Performance of helical ribbon and screw impellers for mixing viscous fluids in cylindrical reactors. Chem. Engineering, 2: 26.

Ameur H, Vial C (2020). Modified scaba 6SRGT impellers for process intensification: Cavern size and energy saving when stirring viscoplastic fluids. Chemical Engineering \& Processing: Process Intensification, 148: 107795.

Ammar M, Abid M S (2011). Numerical investigation of turbulent flow generated in baffled stirred vessels equipped with three different turbines in one and two-stage system. Energy 36, 5081-5093.

Alic F (2013). Thermal-hydraulic irreversibility of electrically heated impeller blades. Chemical Engineering Science, 104: 549-556.

Bouzit M et Benali L, Hachemi M, Bouzit F (2006). CFD simulation of 3D velocity profile of paddle agitator in stirred vessel with highly viscous Newtonian fluid. Journal of Applied Sciences, 6: 2733-2740. 
Driss Z, Karray S, kchaou H, Abid M S (2011). CFD simulation of the laminar flow in stirred tanks generated by double helical ribbons and double helical screw ribbons impellers. Cent. Eur. J. Eng, 1:413-422.

Fort I (2011). On hydraulic efficiency of pitched blade impellers. Chemical Engineering Research and Design, 89: 611-615.

Fort I, Seichter P, Pesl L (2013). Axial thrust of axial flow impellers. Chemical Engineering Research and Design, 91: 789-794.

Foukrach M, Bouzit M, Ameur M, Kamla Y (2019). Influence of the vessel shape on the performance of a mechanically agitated system. Chemical Papers, 73: 469-480.

Hadjeb A, Bouzit M, Kamla Y, Ameur H (2019). A new geometrical model for mixing of highly viscous fluids by combining two-blade and helical screw agitators. Polish Journal of Chemical Technology, 19: 83-91.

Hashimoto S, Natami K, Inoue Y (2009). Experimental study on geometric structure of isolated mixing region in impeller agitated vessel. Chemical Engineering Science, 64: 5173-5181.

Kamla Y, Bouzit M, Ameur H, Arab M I, Hadjeb A (2017). Effect of the inclination of baffles on the power consumption and fluid flows in a vessel stirred by a Rushton turbine. Chin. J. Mech. Eng, 30: 1008-1016.

Kang Q, He D, Zhao N, et al (2019). Hydrodynamics in unbaffled liquid-solid stirred tanks with free surface studied by DEM-VOF method. Chemical Engineering Journal: 122846.

Kazemzadeh A Ein-Mozaffri F, Lohi A, Pakzad L (2017). Intensification of mixing of shearthinning fluids possessing yield stress with the coaxial mixers composed of two different central impellers and an anchor. Chemical Engineering and Processing 111: 101-114.

Laidoudi H, Ameur H (2020). Investigation of the mixed convection of power-law fluids between two horizontal concentric cylinders: Effect of various operating conditions. Thermal Science and Engineering Progress 20: 100731.

Liu B, Zheng Y, Huang B, Qian L, Jin Z (2015) The influence of feeding location on themicro mixing performance of novellarge-double-blade impeller. Journal of the Taiwan Institute of Chemical Engineers, 52: 65-71.

Luan D et al (2017). Effect of the 6PBT stirrer eccentricity and off-bottom clearance on mixing of pseudoplastic fluid in a stirred tank. Results in Physics. 7: 1079-1085.

Martinez-Delgadillo S A et al (2019). Analysis of the turbulent flow and trailing vortices induced by new design grooved blade impellers in a baffled tank. Chemical Engineering Journal. 358: 225-235.

Moucha T et al (2003). Gas hold-up, mixing time and gas-liquid volumetric mass transfer coefficient of various multiple-impeller configurations: Rushton turbine, pitched blade and techmix impeller and their combinations. Chemical Engineering Science, 58: 1839 - 1846.

Rajasekaran E et al (2018). Determination of forced convection heat transfer coefficients and development of empirical correlations for milk in vessel with mechanical agitators. Journal of Food Science Technology, 55: 2514-2522.

Ramírez-Muñoz $\mathbf{J}$ et al (2016). Assessment of the effective viscous dissipation for deagglomeration processes induced by a high shear impeller in a stirred tank. Advanced Powder Technology, 27: 1885-1897.

Su T, Yang F, Li M and Wu K (2018). Characterization on the Hydrodynamics of a Coveringplate Rushton impeller. Chinese Journal of Chemical Engineering, 26: 1392-1400.

Tacay C D, Payunescu M (2000). Suspension of solid particles in spherical stirred vessels. Chemical Engineering Science, 55: 2989-2993.

Tamburini A, Cipollina A, Micale G, Brucato A and Ciofalo M (2012). CFD simulations of dense solid-liquid suspensions in baffled stirred tanks: Prediction of the minimum impeller speed for complete suspension. Chemical Engineering Journal, 193-194: 234-255.

Vakili M H, Nasr Esfahany M (2009). CFD analysis of turbulence in a baffled stirred tank, a three-compartment model. Chemical Engineering Science, 64: 351 - 362. 
Vilard G, Verdone N (2019). Production of metallic iron nanoparticles in a baffled stirred tank reactor: Optimization via computational fluid dynamics simulation. Particuology.

Youcefi A (1993). Etude expérimentale de l'écoulement de fluide viscoélastique autour d'un agitateur bipale dans une cuve agitée. (Experimental study of viscoelastic fluid flow around two-blade impeller in a stirred vessel), PhD Thesis, Ecole Nationale Polytechnique (National Polytechnic Institute), Toulouse, (in French). 